\title{
Whitening in Range to Improve Weather Radar Spectral Moment Estimates. Part II: Experimental Evaluation
}

\author{
IGOR R. IVIĆ \\ Cooperative Institute for Mesoscale Meteorological Studies, University of Oklahoma, and \\ National Severe Storms Laboratory, Norman, Oklahoma \\ DUŠAN S. ZRNIĆ \\ National Severe Storms Laboratory, Norman, Oklahoma \\ SEbASTIÁN M. TORRES \\ Cooperative Institute for Mesoscale Meteorological Studies, University of Oklahoma, and \\ National Severe Storms Laboratory, Norman, Oklahoma
}

(Manuscript received 10 September 2002, in final form 17 March 2003)

\section{ABSTRACT}

\begin{abstract}
Demonstration of a method for improved Doppler spectral moment estimation is made on NOAA's research and development Weather Surveillance Radar-1988 Doppler (WSR-88D) in Norman, Oklahoma. Time series data have been recorded using a commercial processor and digital receiver whereby the sampling frequency is several times larger than the reciprocal of the transmitted pulse width. The in-phase and quadrature-phase components of oversampled weather signals are used to estimate the first three spectral moments by suitably combining weighted averages in range with usual processing at fixed range locations. The weights are chosen in such a manner that the resulting signals become uncorrelated. Consequently, the variance of estimates decreases significantly as is verified by this experiment.
\end{abstract}

\section{Introduction}

There are compelling scientific and practical reasons to rapidly acquire volumetric radar data. For example, observations at minute intervals are required to understand the details of vortex formation and demise near the ground. Even faster rates of volumetric data are needed to determine the presence of transverse winds (Shapiro et al. 2001). Fast update rates would also yield more timely warnings of impending severe weather phenomena such as tornadoes and strong winds. Even rainfall measurement would be better if radar data were available at shorter intervals than the current 6 min (Fabry et al. 1994).

Surveillance weather radars have a mechanical control of beam position, and dwell a relatively long time $(\sim 50 \mathrm{~ms})$ to obtain a sufficient number of independent echo samples for accurate estimates of Doppler spectral moments (Doviak and Zrnić 1993). Thus, the volume update times are dictated by two limitations: 1) the inertia of the mechanically steered antenna, and 2) the

Corresponding author address: Igor Ivić, NSSL, 1313 Halley Circle, Norman, OK 73069.

E-mail: igor.ivic@noaa.gov correlation time of weather signals. Phased array radars promise to increase the speed of volume coverage. Reports about simultaneous use of phased array radar for tactical application and weather observation indicate that a radial of data can be obtained from only two transmitted pulses as opposed to over 40 on the Weather Surveillance Radar-1988 Doppler (WSR-88D; Owen et al. 1998). Closer examination of this success reveals that rapid beam swinging is not the main contributor to such fast updates; rather, it is the pulse compression. Pulse compression requires wide transmitter bandwidth that is not available to the operational weather radar community, hence, the dilemma of how to increase the data acquisition speed and fully utilize the rapid beam steering afforded on phased array radars. An improvement by a factor of 2 is possible by "beam multiplexing," where the data from different directions are collected in a repetitive time-shared pattern (Doviak et al. 2001). This, however, falls short of the desired capability.

A very different scheme to process weather radar data has recently been proposed. Koivunen and Kostinski (1999) suggest to decorrelate (whiten) the time series of weather echos and then process these to obtain better 

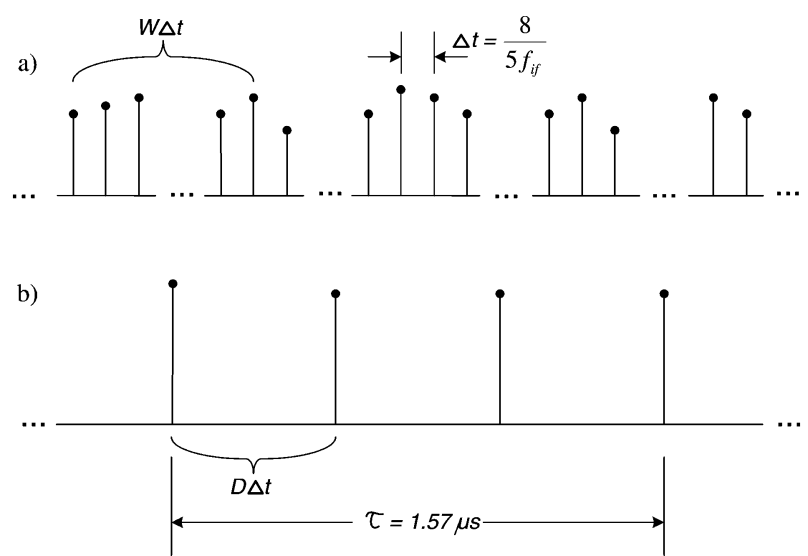

FIG. 1. Samples of (a) digitized IF signal and (b) base-band $I$ and $Q$ signal for the short pulse in an RVP7.

estimates of powers. To achieve this type of whitening, prior knowledge of the weather signal correlation coefficient is required (Schultz and Kostinski 1997). Consequently, the spectrum should be known or rather tight constraints on the range of its values must be imposed, neither of which is practical. These issues are discussed in the cited and other references (Kostinski and Koivunen 2000).

Torres and Zrnić (2003) capitalize on the known be-
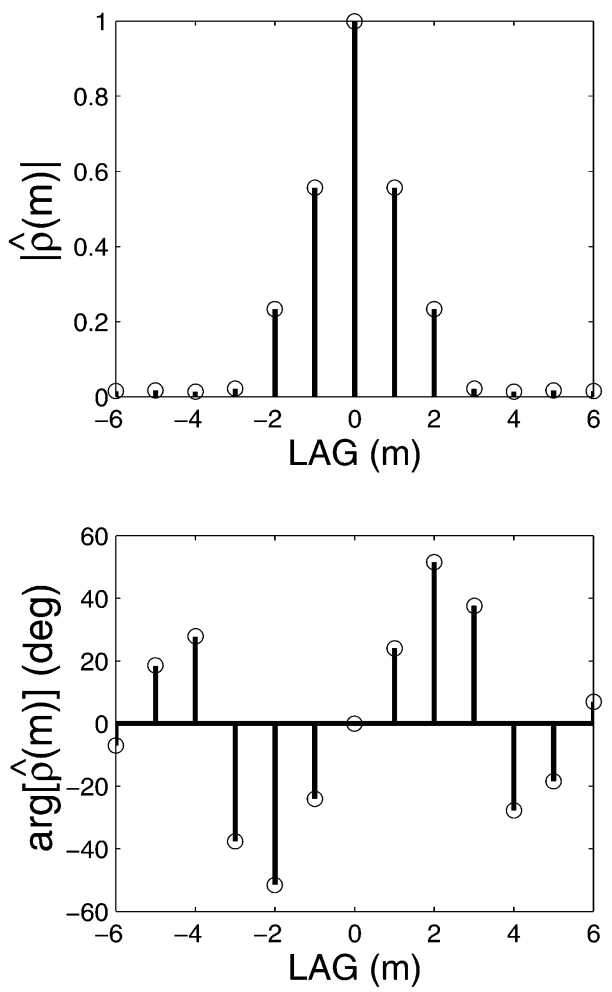

a) havior of weather signals in range and present a viable method for reducing variance of spectral moment estimates. They propose to sample the signal in range at time intervals $L$ times smaller than the pulse duration. Then, by a linear transformation, they whiten the $L$ samples, compute various autocovariances (or Doppler spectra) of the samples, and combine these to obtain spectral moment estimates. The underpinning assumption is that the oversampled weather signals are widesense stationary over the pulse depth. This assumption is implicit in standard processing of weather signals whereby a matched filter precedes spectral processing. An in-depth analysis of the scheme is documented elsewhere (Part I of this paper; also Torres 2001), whereas herein we present a sample of a test on the National Oceanic and Atmospheric Administration (NOAA) research and development WSR-88D radar to demonstrate its practicality.

\section{Theoretical background}

If the returned echoes are sampled more frequently in range than once per pulse duration, it is possible to reduce the variances of estimates. Averaging of $L$ oversampled estimates reduces the variance by a factor $L_{I}$ known as the effective number of independent samples, which is computed from
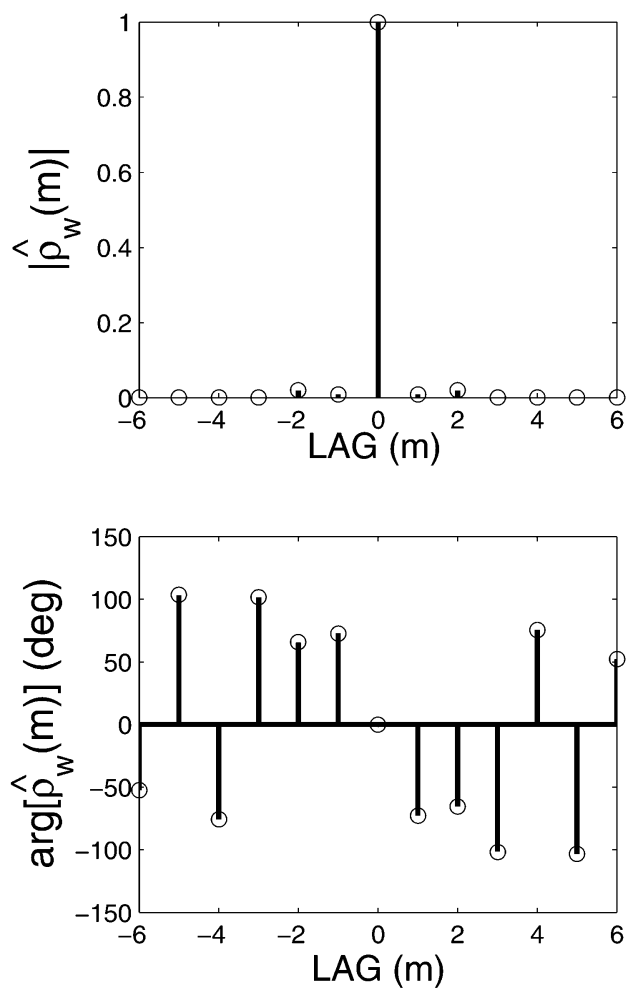

b)

FIG. 2. Magnitude and argument of the autocorrelation (a) before and (b) after whitening for $L=3$, obtained from time series of weather data. The short pulse was transmitted and one lag corresponds to $\sim 0.57 \mu$ s. 

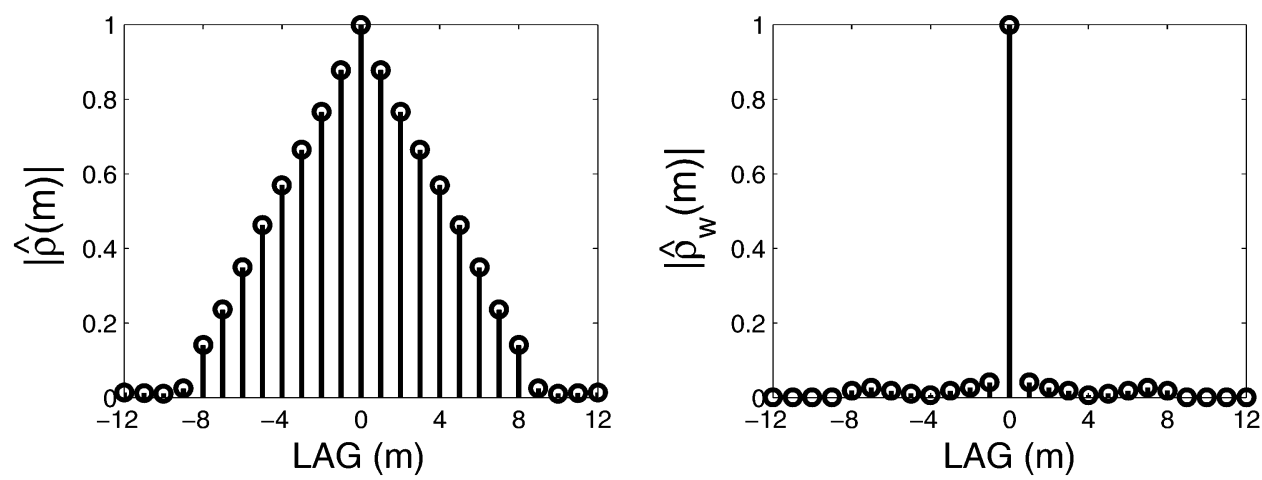

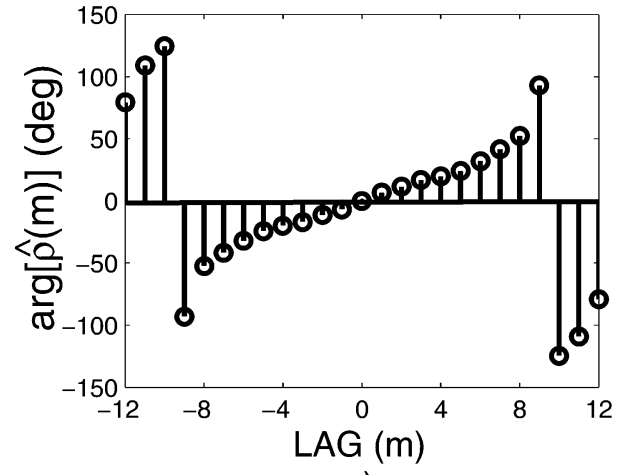

a)

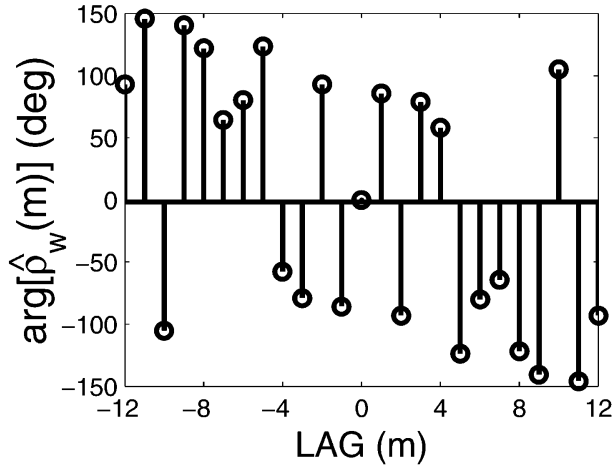

b)

FIG. 3. Magnitude and argument of the autocorrelation (a) before and (b) after whitening for $L=9$, obtained from time series of weather data. The transmitted pulse was long and one lag corresponds to $\sim 0.57 \mu$ s.

$$
L_{I}^{-1}=\sum_{l=-(L-1)}^{L-1} \frac{L-|l|}{L^{2}}\left|\rho_{S}(l)\right|,
$$

where $\rho_{S}(l)$ is the correlation coefficient of the variable averaged to obtain the estimates (Doviak and Zrnić 1993). Note that the maximum possible reduction in variance through averaging is by $L$, which is achieved only if signals are uncorrelated. Thus, if samples are

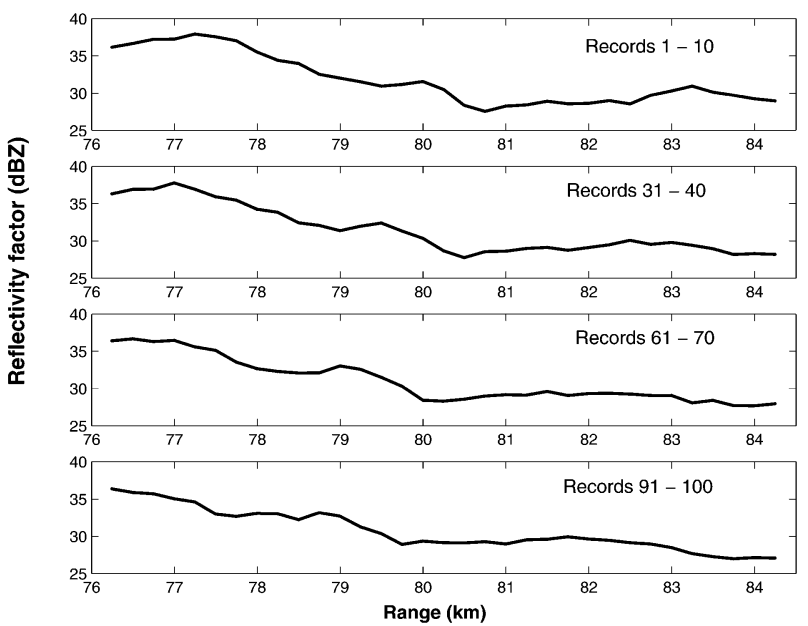

FIG. 4. Estimates of reflectivity factor on sets of 10 records spaced $20 \mathrm{~s}$ apart, using an oversampling factor $L=3$ (SNR $>35 \mathrm{~dB}$ ). correlated, simple averaging does not lead to an optimum variance reduction. As an example, consider a rectangular transmitted pulse and a receiver with infinite bandwidth for which the magnitude of the correlation coefficient of oversampled complex signals in range is (Doviak and Zrnić 1993)

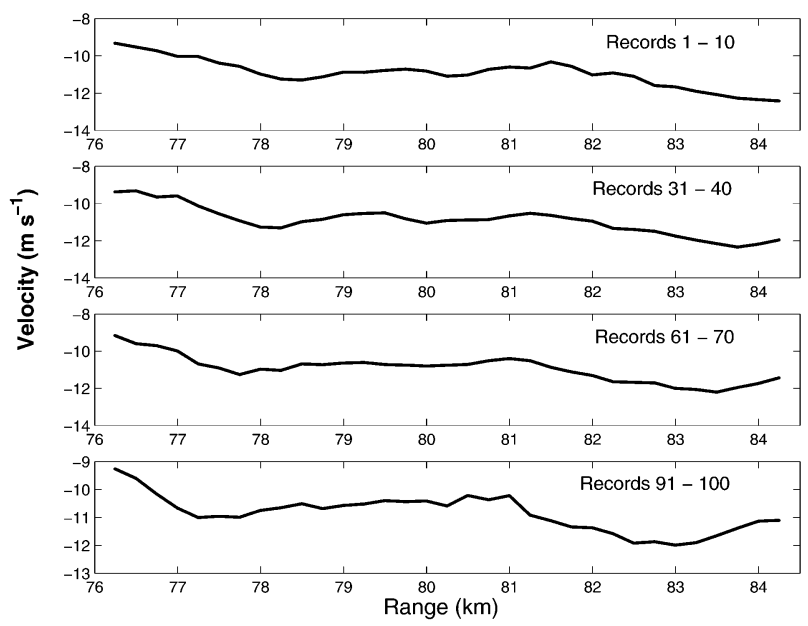

FIG. 5. Estimates of mean Doppler velocity on sets of 10 records spaced $20 \mathrm{~s}$ apart, using an oversampling factor $L=3$ (SNR > $35 \mathrm{~dB})$. 


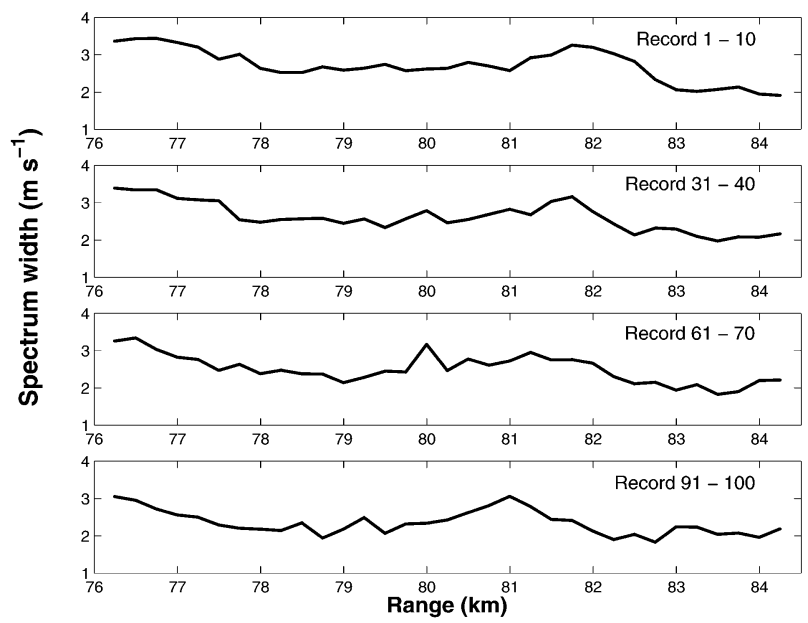

FIG. 6. Estimates of Doppler spectrum width on sets of 10 records spaced $20 \mathrm{~s}$ apart, using an oversampling factor $L=3$ (SNR $>35 \mathrm{~dB})$.

$$
\rho(l)= \begin{cases}1-|l| / L & |l|<L \\ 0 & \text { otherwise } .\end{cases}
$$

The correlation coefficient for oversampled powers is $|\rho(l)|^{2}=\left|\rho_{S}(l)\right|$. Substituting this $\rho_{S}(l)$ in (1), one finds that $L_{I}=2$ in the limit for very large $L$. Therefore,

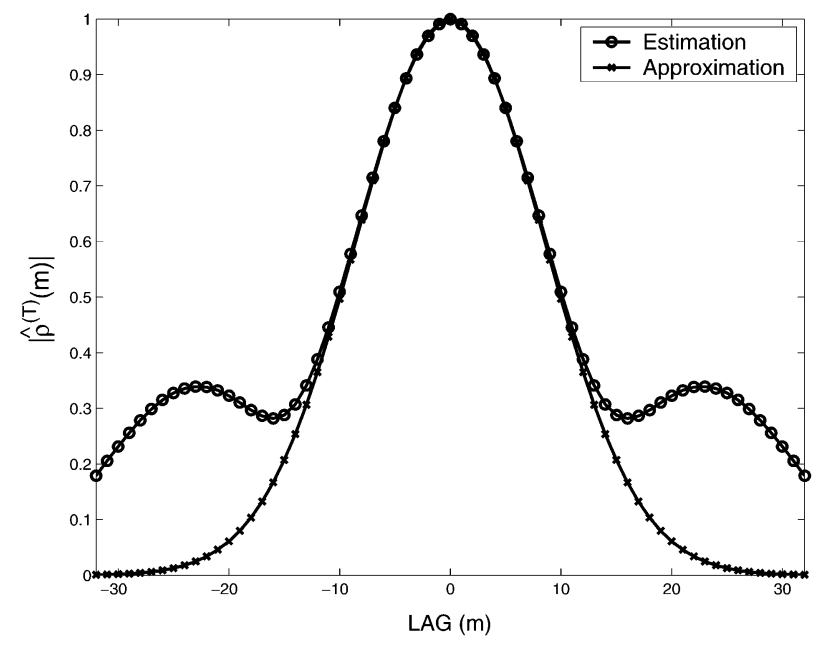

FIG. 7. Estimated $(\bigcirc)$ and approximated $(\times)$ autocorrelation coefficient along sample time for the difference threshold set at $30 \%$.

a reduction of at most 2 in variance is possible by directly averaging the oversampled power estimates.

A different scheme to process the samples and achieve a significantly smaller variance of estimates is described in Part I of this paper (Torres and Zrnić 2003, hereafter Part I). It entails decorrelating the oversampled

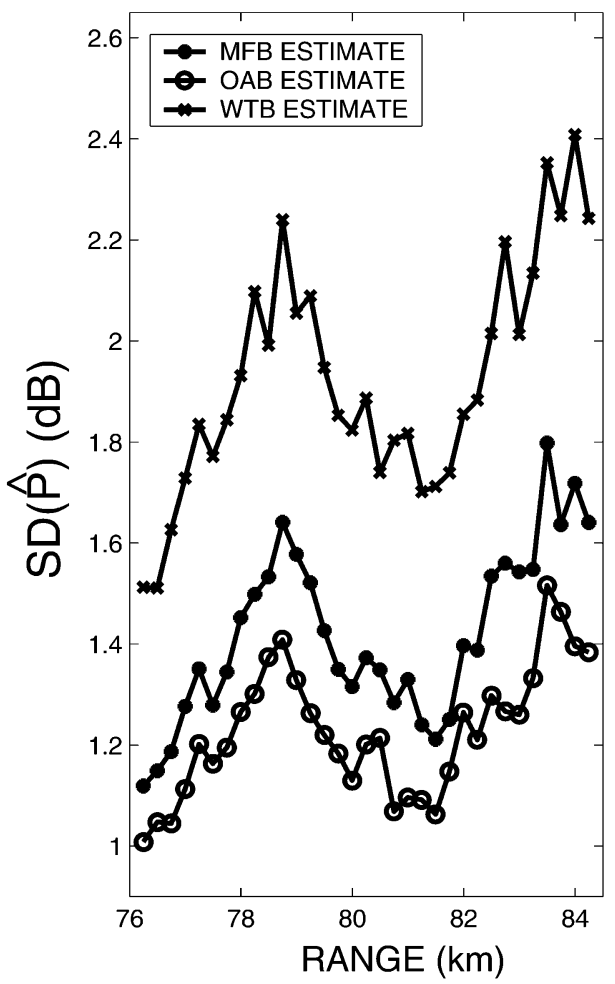

a)

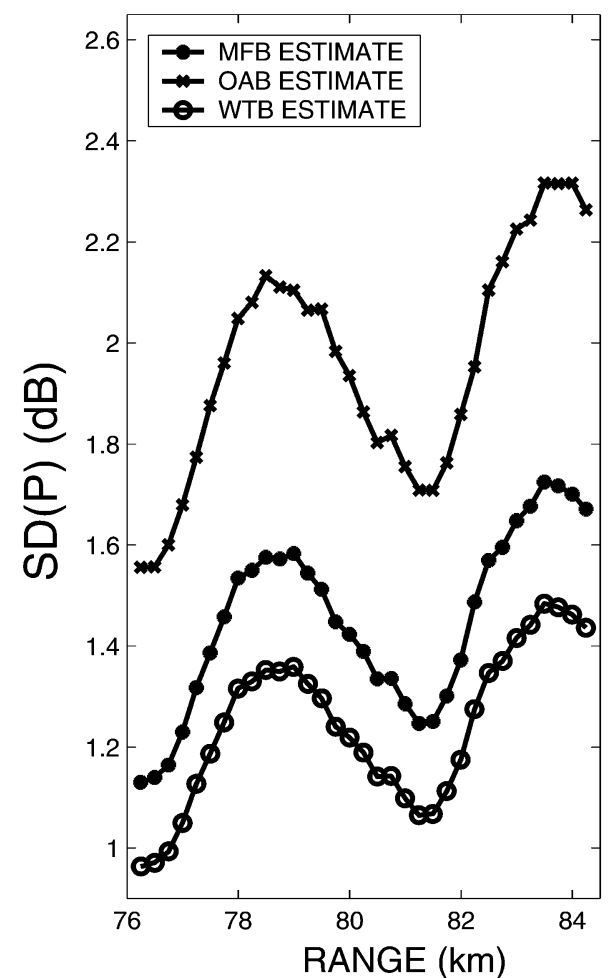

b)

FIG. 8. (a) Experimental and (b) theoretical normalized std dev of power estimates in $\mathrm{dB}$ for an oversampling factor of $L=3$. 


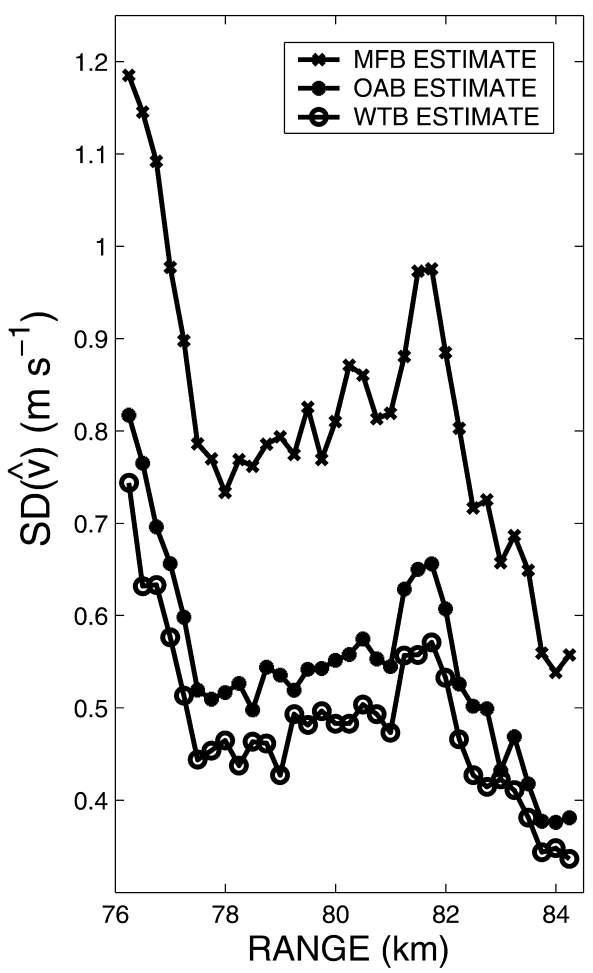

a)

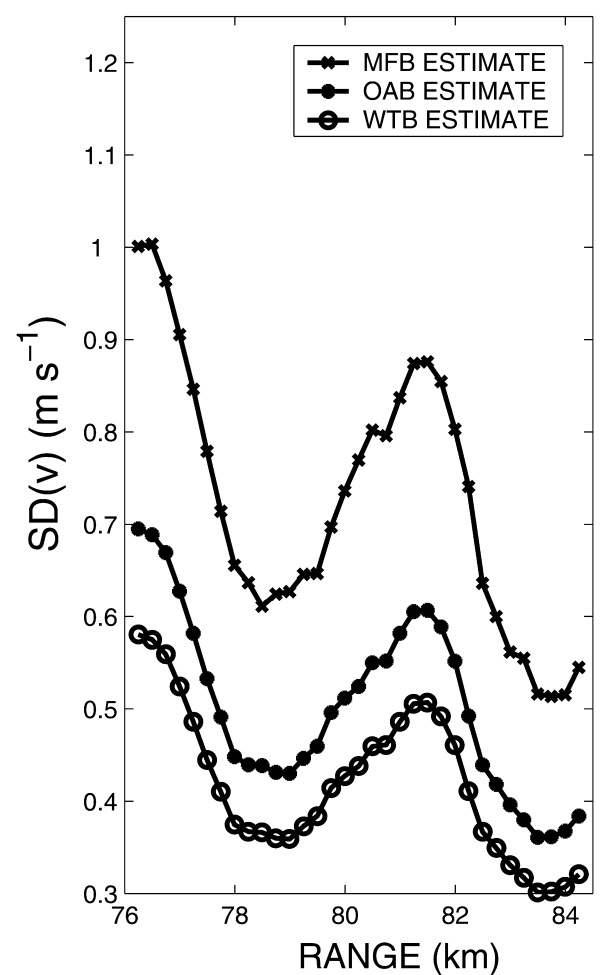

b)

FIG. 9. (a) Experimental and (b) theoretical std dev of velocity estimates for an oversampling factor of $L=3$.

signals in range and then processing these in the usual manner. That is, powers of the uncorrelated signals (in range) are averaged to obtain the reflectivity, and estimates of the autocovariance in sample time are averaged to retrieve the mean Doppler velocity and spectrum width.

If the reflectivity is uniform on a distance of two pulse depths, the correlation coefficient of oversampled signals along range time is a function of the pulse shape and the receiver impulse response (Doviak and Zrnić 1993). This range-time correlation coefficient can be measured on data or by passing the (attenuated) transmitted pulse through the receiver and recording the response. It can then be used to whiten the samples as described in the formulation of Part I.

It is instructive to compare the variances of estimates obtained by averaging in range autocovariances of oversampled signals with those obtained from averages of whitened signals. If noise effects are not taken into consideration, in the case of a rectangular pulse, the ratio of the two variances is

$$
\frac{\operatorname{var}\left(E_{\text {correlated }}\right)}{\operatorname{var}\left(E_{\text {whitened }}\right)}=\frac{L^{2}+1}{2 L},
$$

where $E_{\text {correlated }}$ stands for the estimate obtained by averaging $L$ autocovariances from oversampled signals and $E_{\text {whitened }}$ is the estimate obtained by averaging $L$ au- tocovariances from whitened oversampled signals (Torres 2001).

\section{Experimental setup}

For validation of the theoretically predicted variance reductions we used the SIGMET RVP7 digital processor passively coupled to the research and development WSR-88D weather radar in Norman, Oklahoma. The RVP7 incorporates a digital receiver at intermediate frequency (IF) and has an inherent capability to sample signals at higher rates than the reciprocal of the pulse width. In addition, it can record a limited amount of time series data ( $I$ and $Q$ ). An antialias filter centered on the intermediate frequency $\left(f_{\text {if }} \sim 57 \mathrm{MHz}\right)$ and with a bandwidth of about $8 \mathrm{MHz}$ precedes the digital receiver. The IF signal was tapped ahead of the existing matched filter at a point where the receiver bandwidth was about $15 \mathrm{MHz}$ and was passed through the antialias filter. Hence, the frequency response at the input to the digital receiver is primarily shaped by the antialias filter. The WSR-88D can easily switch between two pulse lengths: short equal to $1.57 \mu \mathrm{s}$ (250-m range resolution) and long equal to $4.71 \mu$ s (750-m range resolution). The scheme was tested on both of these.

Constraints on the RVP7 around which we designed the experiment are as follows. The sampling frequency 


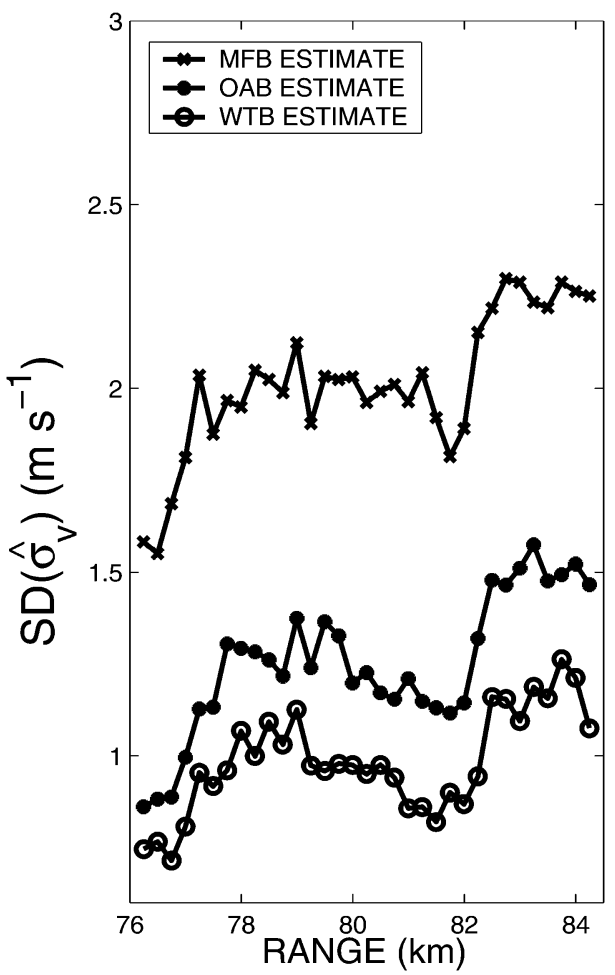

a)

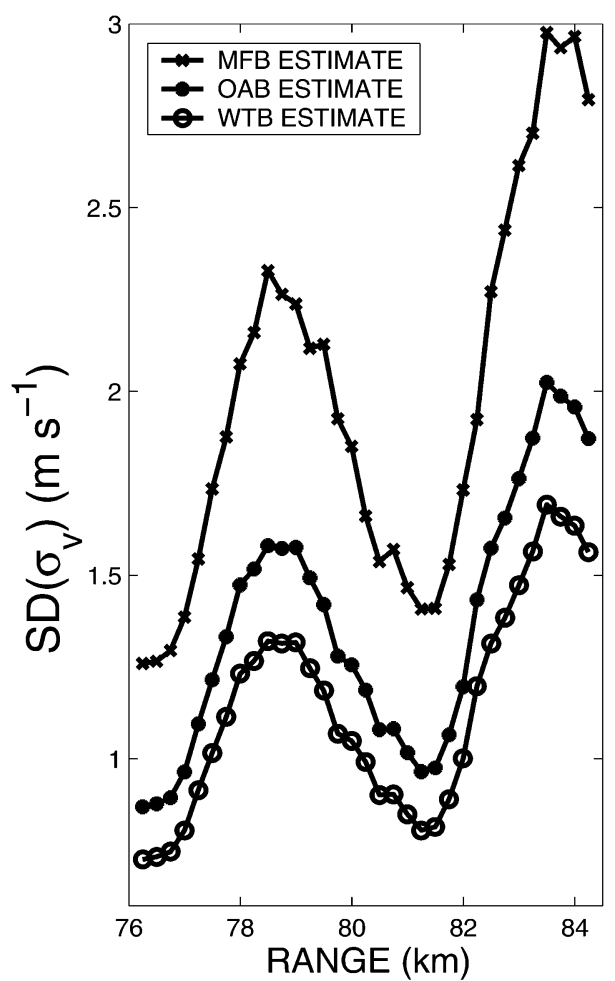

b)

FIG. 10. (a) Experimental and (b) theoretical std dev of Doppler spectrum width estimates for an oversampling factor of $L=3$.

$f_{s}$ for the intermediate-frequency $\left(f_{\text {if }}\right)$ signal is $(5 / 8) f_{\mathrm{if}}$. After sampling, a digital downconversion is combined with a finite impulse response (FIR) filter (SIGMET 1999). The filter has a variable number of taps $W$, and the spacing of output digital samples is $D / f_{s}$ which is also variable (Fig. 1). The weights on the FIR filter can be programmed for a desired frequency response; we chose a uniform set. The digital sinusoid for downconversion starts with the same phase every $D / f_{s}$ seconds and therefore has a variable phase relation (from sample to sample) with respect to the IF signal unless $D$ is a multiple of five. It is not possible to set $D$ to an odd number, and the minimum allowable even multiple of five for $D$ is 20 . Phase discontinuities among output range samples render the whitening algorithm ineffective; thus, we chose $D=20$ (corresponding to gate spacing of $83.3 \mathrm{~m}$ ) and the same value was used for the number of taps $W$. With this $D$, the number of samples $L$ is three within the short pulse and nine within the long pulse.

As mentioned before, the processor can record time series data $(I$ and $Q)$ from a limited number of range gates. In the experiment we recorded data from 101 consecutive range locations spaced $83.3 \mathrm{~m}$ apart; each set of 101 samples from one transmitted pulse form a radial, and 128 radials constitute one record (due to the constraints imposed by RVP7 there is a gap of about 1 $\mathrm{s}$ between records).

\section{Measurements and analysis of results}

Several sets of data from weather events and ground clutter were recorded in both the short (regular) and the long pulse mode. The whitening-transformation-based (WTB) estimates were obtained from these datasets.

\section{a. Correlation coefficient}

Implementation of the whitening transformation requires knowledge of the autocorrelation function of oversampled signals along range. One robust way to compute the set of autocorrelation coefficients is from the oversampled weather data themselves. The magnitudes and phases of the correlations obtained in this manner are given in Figs. 2 and 3 for both pulse lengths. These curves are averages of sample autocorrelation coefficients in both range and time. The number of range locations for averaging was $\lfloor 101 / L\rfloor$; the number of averages in time was 1280 (i.e., 1280 samples were averaged at each lag). At every range location the signalto-noise ratio (SNR) was larger than $35 \mathrm{~dB}$. For both pulse lengths the magnitude of the autocorrelation (Figs. 2a, 3a) has a fairly triangular shape, which is expected from a rectangular pulse. The deviation from straight lines is likely due to the nonvertical leading and trailing edges of the pulse and the effects of the overall radar system filter. The correlation coefficient of samples was 


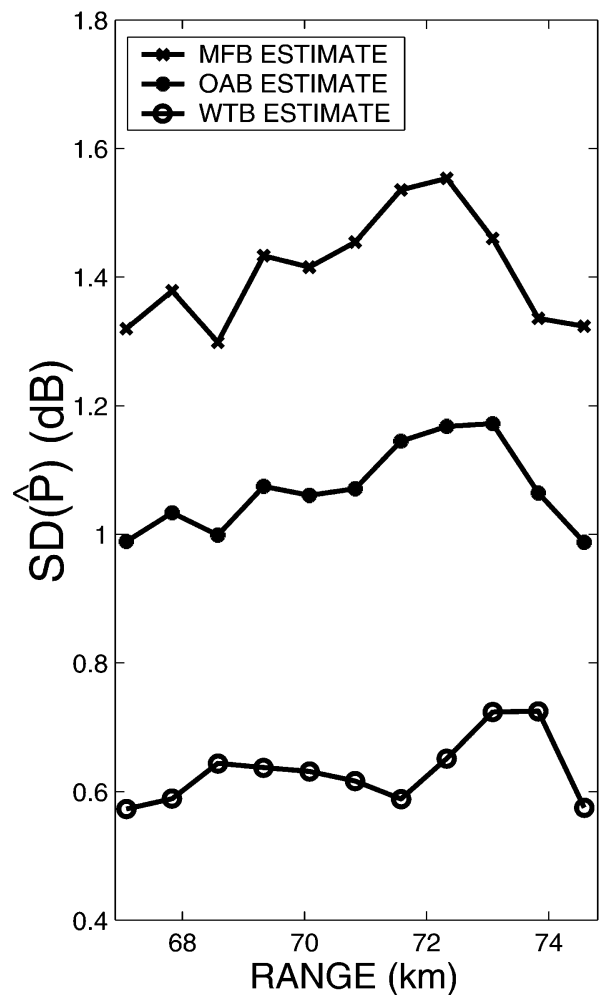

a)

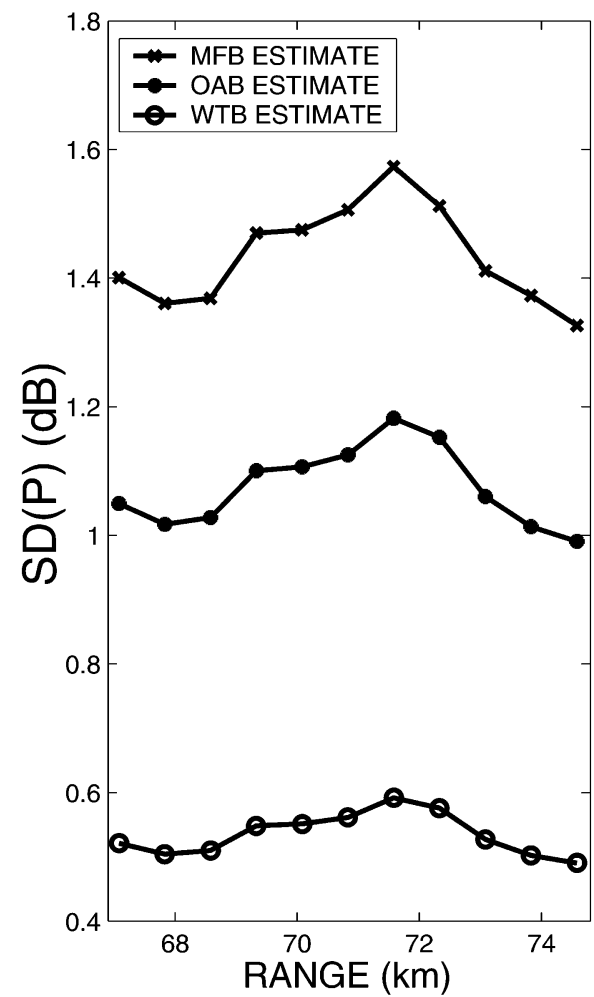

b)

FIG. 11. (a) Experimental and (b) theoretical normalized std dev of power estimates in $\mathrm{dB}$ for an oversampling factor of $L=9$.

also computed after the application of the whitening transformation [Eq. (3), Part I]. The result is a noise like peak at lag zero and insignificant values at other lags (Figs. 2b, 3b).

We draw attention to the phases of the correlation coefficients. Note that the phase is nontrivial for the nonzero values of the magnitude. This phase shift is caused by the amplitude modulation (AM) to phase modulation (PM) conversion whereby the voltage variations of the transmitted pulse envelope (primarily rise and decay, and secondarily small ripples) are converted into phase variations of the radio frequency (RF) carrier. This effect is inherent to the klystron amplifier and it was found to be repetitive.

\section{b. Spectral moments}

Mean power, mean Doppler velocity, and Doppler spectrum width were computed using estimators given in Part I [Eqs. (14), (16), and (19)] in the following three ways.

1) They were computed from regularly spaced range locations (at $83.3 \mathrm{~m}$ ) first by averaging in range the in-phase and quadrature-phase $(I, Q)$ components, and then by processing the autocovariances to produce the moments. The coherent averaging of samples over the pulse duration (three samples for short pulse and nine for long pulse) is a digital matched filter that approximates its analog counterpart fairly well. Henceforth, this procedure is called the matched-filter-based (MFB) processing.

2) They were computed from averages of autocovariances of oversampled signals. The number of averages in range was three for the short pulse and nine for the long pulse. We call this procedure the oversampling-and-average-based (OAR) processing. It is a straightforward extension of the MFB processing, and about the best one can achieve with correlated oversampled data.

3) They were computed from averages of autocovariances of oversampled and whitened data. The same number of averages as in 2) was used for the whitening-transformation-based (WTB) processing.

Each initial autocovariance estimate (including at lag zero for power) was obtained from 32 contiguous time samples ( $I$ and $Q$ components), which corresponded to dwell times of $31.57 \mathrm{~ms}$ for the short pulse and 71.68 $\mathrm{ms}$ for the long pulse. The pulse repetition frequencies were $1013.51 \mathrm{~Hz}$ for the short pulse and $446.43 \mathrm{~Hz}$ for the long pulse.

For the real data used in the experiment the true values of estimates are not known but are required for calculation of variances. Consequently, these "true" values had to be estimated as well. To achieve this, short-term 


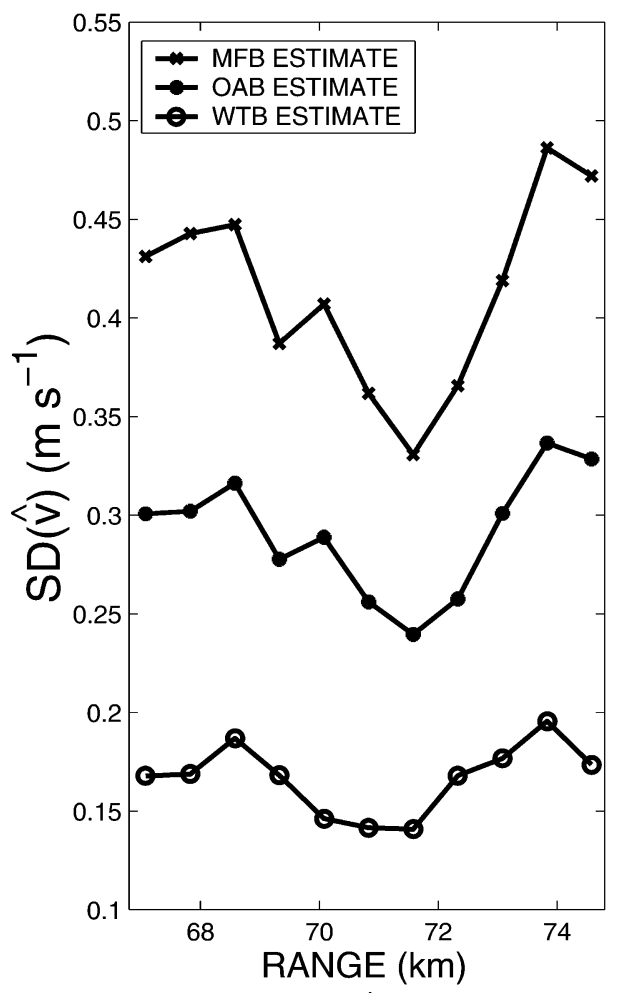

a)

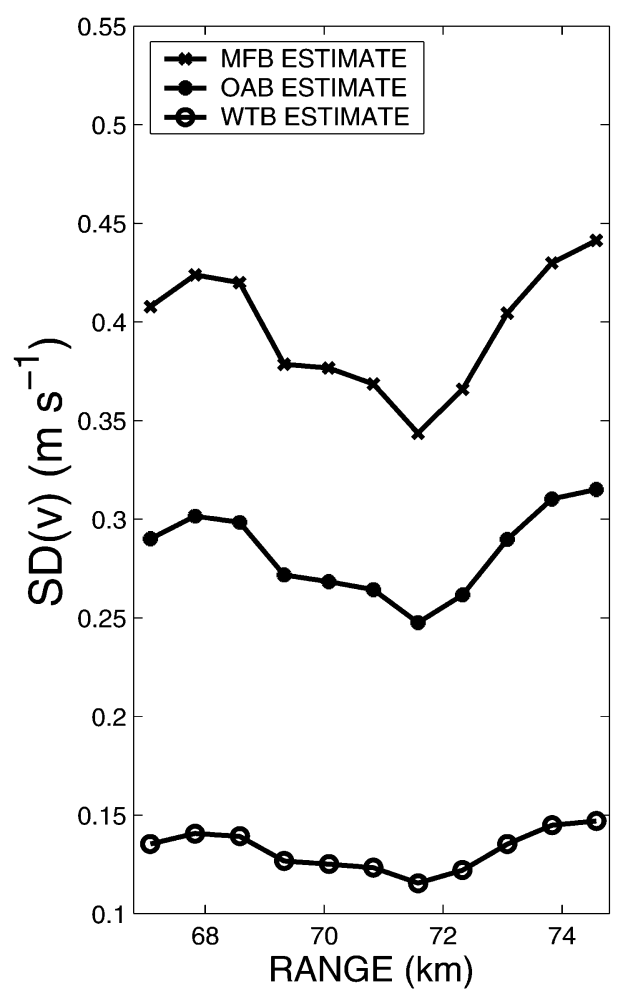

b)

Fig. 12. (a) Experimental and (b) theoretical std dev of velocity estimates for an oversampling factor of $L=9$.

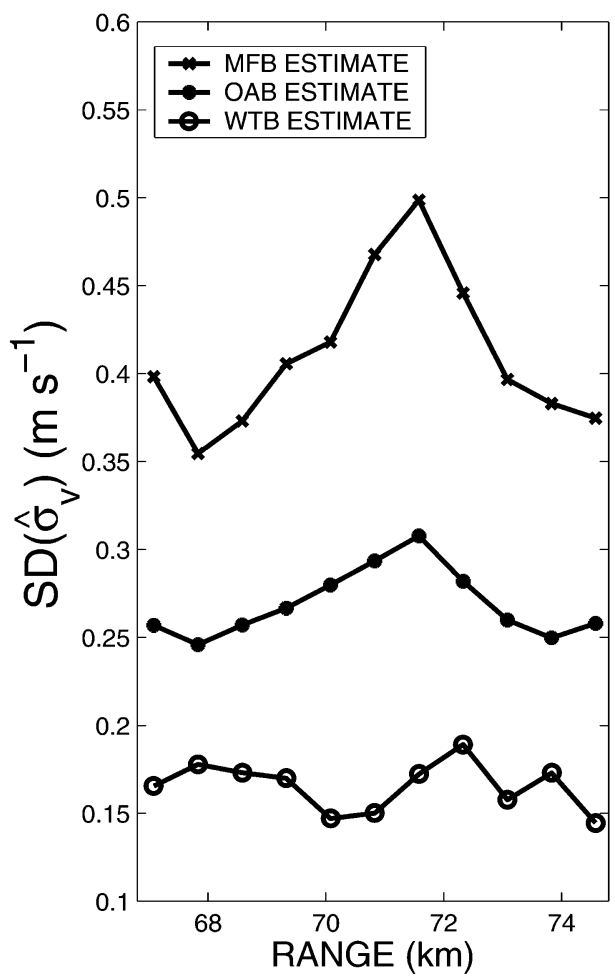

a)

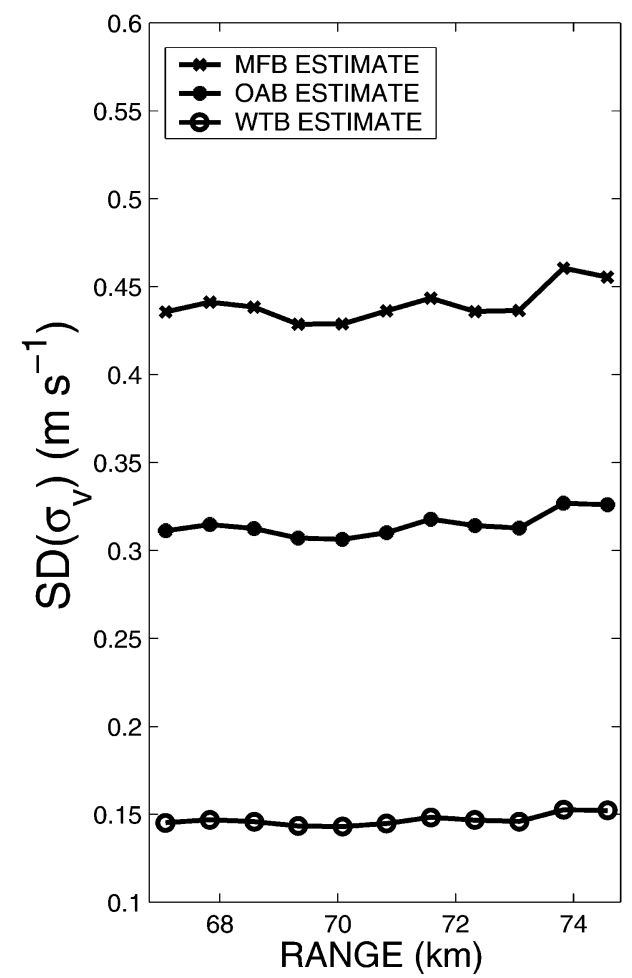

b)

FIG. 13. (a) Experimental and (b) theoretical std dev of Doppler spectrum width estimates for an oversampling factor of $L=9$. 


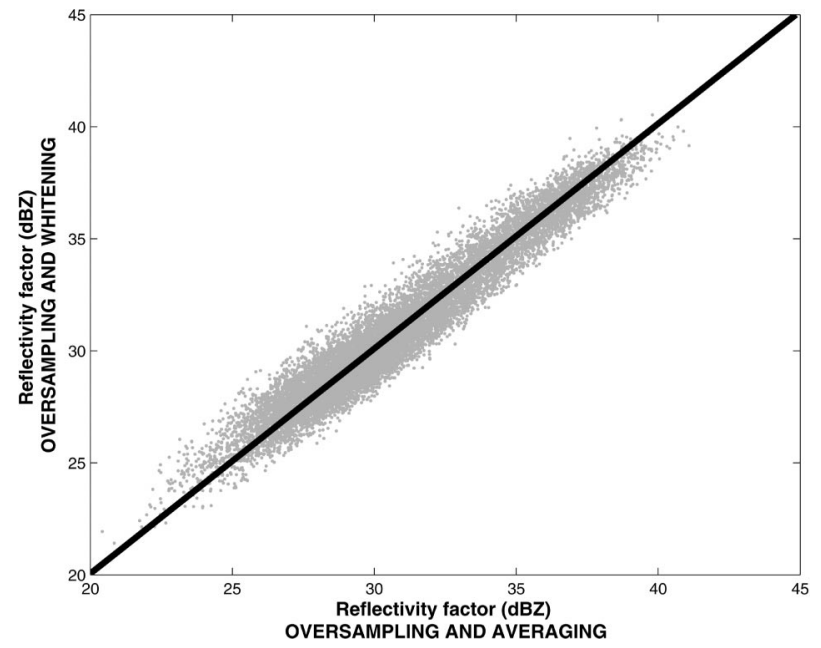

FIG. 14. Scatterplot of WTB vs OAB power estimates. The solid line is the best fit in the least squares sense.

(local) estimates of the mean values of autocovariances and corresponding spectral moments were obtained from 10 consecutive records of data. Because we had 100 records, 10 variances at each range location obtained from local mean estimates were averaged, and their square roots (standard deviations) are plotted herein for comparisons. Short-term averaging was necessary to mitigate the effects of radial changes (in the structure of the spectral moments) due to advection and evolution. Plots of the spectral moments versus range for records spaced $20 \mathrm{~s}$ apart indicate progressive systematic changes in details (Figs. 4, 5, and 6, obtained from OAB estimates). The reflectivity features (Fig. 4) have clearly advected toward the radar by about $1 \mathrm{~km}$ during the time of 111 s (i.e., 100 records). Similar advection is present in the other two spectral moments. Advection across the beam and evolution are likely the cause of other changes in these features.

There are different ways to deal with the fact that the correlation coefficient is complex (attributed to the phase shift produced by the AM-to-PM conversion in the klystron amplifier). One is to use it directly in Eq. (4) of Torres and Zrnić (2003). The other is to take out the progressive phase shift within the pulse that the AMto-PM conversion causes, and then use the magnitude of the correlation coefficient in the aforementioned equation to decorrelate the samples. We opted for the latter.

We paired the plots of estimated standard deviations with "theoretical" values of standard deviations at each range location and for each procedure. By theoretical we mean prediction from analytic formulas and derivations applied thereof. The analytic formulas can be found in Doviak and Zrnić 1993 [Eqs. (6.10), (6.21), and (6.30a)] along with the conditions under which those are valid [Eqs. (6.20a) and (6.20b)]. They contain summations of correlation coefficient at various lags, as well as noise effect terms. The sample-time autocor-

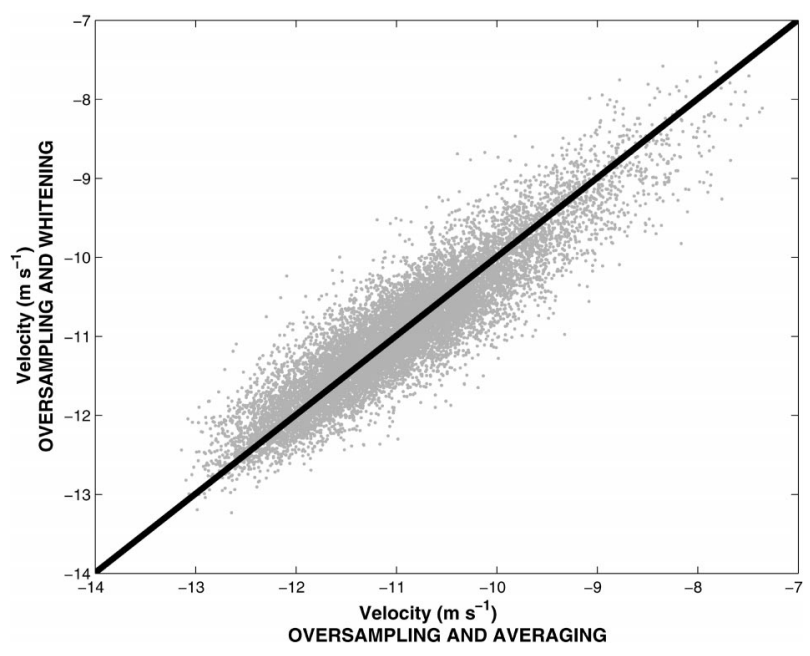

FIG. 15. Scatterplot of WTB vs OAB velocity estimates. The solid line is the best fit in the least squares sense.

relation coefficient was obtained by fitting a Gaussian shape to the autocorrelation estimated from data. Because the accuracy of estimates is inversely proportional to the lag number, an adaptive fit was used. It starts from the peak of the correlation estimate (at lag zero) and proceeds to smaller values (larger lag values) dynamically averaging the width of the fit to closely match the observed autocorrelation shape. The process stops when the difference between the fitted and estimated correlation is larger than a threshold of 30\% (Fig. 7). This determined the value of the width, which was then used to calculate the autocorrelation at subsequent lags. In all of our examples the SNR was larger than $35 \mathrm{~dB}$; hence, the noise enhancement effects were negligible (Torres and Zrnić 2003). Consequently, the noise was set to zero in theoretical computations. The standard

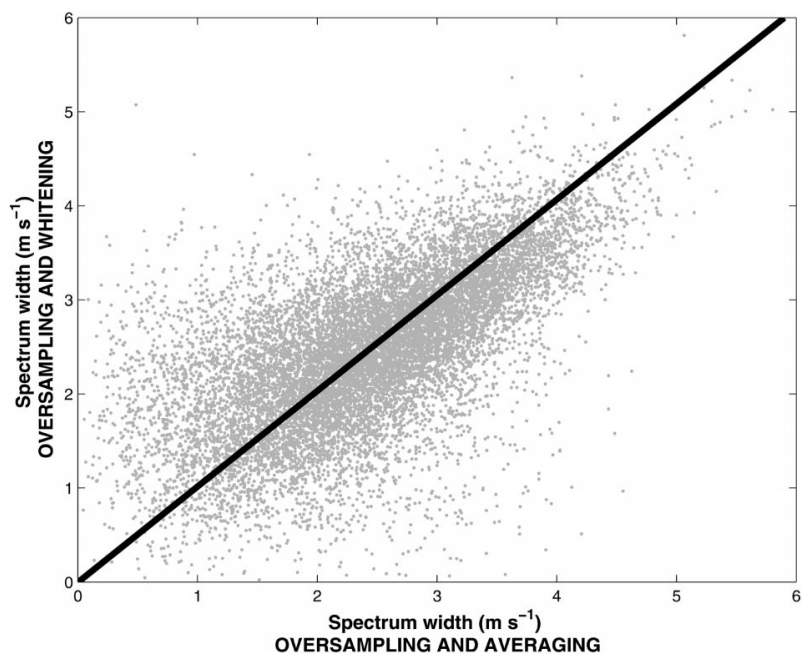

FIG. 16. Scatterplot of WTB vs OAB spectrum width estimates. The solid line is the best fit in the least squares sense. 
TABLE 1. Variance ratios obtained for $L=3$. The acronyms in the subscript are defined in text; subscript " $\mathrm{t}$ " stands for theoretical.

\begin{tabular}{lccccc}
\hline \hline & $\frac{\operatorname{var}\left(\hat{X}_{\mathrm{MFB}}\right)}{\operatorname{var}\left(\hat{X}_{\mathrm{OAB}}\right)}$ & $\frac{\operatorname{var}\left(\hat{X}_{\mathrm{MFBt}}\right)}{\operatorname{var}\left(\hat{X}_{\mathrm{OABt}}\right)}$ & $\frac{\operatorname{var}\left(\hat{X}_{\mathrm{MFB}}\right)}{\operatorname{var}\left(\hat{X}_{\mathrm{WTB}}\right)}$ & $\frac{\operatorname{var}\left(\hat{X}_{\mathrm{MFBt}}\right)}{\operatorname{var}\left(\hat{X}_{\mathrm{WTBt}}\right)}$ & $\frac{\operatorname{var}\left(\hat{X}_{\mathrm{OAB}}\right)}{\operatorname{var}\left(\hat{X}_{\mathrm{WTB}}\right)}$ \\
\hline$X=P$ & 2.105955 & 2.073276 & 3.071418 & 2.970985 & 1.436999 \\
$X=v$ & 2.238192 & 2.082410 & 2.942816 & 2.983750 & 1.317445 \\
$X=\sigma_{v}$ & 2.791660 & 2.092170 & 4.655921 & 2.998174 & 1.432994 \\
\hline
\end{tabular}

deviation $(\mathrm{SD})$ of power estimates was converted to a decibel scale by the equation

$$
\mathrm{SD}(\hat{P})=10 \log \left[1+\frac{\sqrt{\operatorname{var}(\hat{P})}}{P_{o}}\right],
$$

where $\hat{P}$ is the power estimate and $P_{o}$ is the estimate of the local mean value of power. Figures 8,9 , and 10 illustrate the standard deviations of spectral moments as a function of range for the oversampling factor $L=3$.

The standard deviation of normalized power estimates obtained from whitened samples is consistently smaller than its counterparts (Fig. 8). All these estimates exhibit maxima at approximately 79 and $83.5 \mathrm{~km}$. It is at these range locations that the spectrum width (see Fig. 6) exhibits a local minima. The spectrum width formula (Doviak and Zrnić 1993) shows that a lower spectrum width corresponds to a higher autocorrelation in sample time. Therefore, the signal autocorrelation along sample time reaches its local maxima at these ranges. Consequently, this decreases the equivalent number of independent samples $\left(L_{I}\right)$ resulting in a higher variance for power estimates. Although whitening has decreased the $\mathrm{SD}(\hat{P})$ the maximum value of about $1.5 \mathrm{~dB}$ is still larger than the 1-dB WSR-88D specification for a $1-\mathrm{km}$ average. If the oversampling factor is increased to 8 (possible but not available on the RVP7 processor), the $\operatorname{SD}(\hat{P})$ over the $250-\mathrm{m}$ pulse depth would be below the $1-\mathrm{dB}$ specification. Note that theoretical values of SD (Fig. 8b) are in good agreement with the experimental data in Fig. 8a.

The experimentally determined SDs of mean Doppler velocity estimates (Fig. 9a) are in reasonable agreement with the corresponding theoretical curves given in Fig. 9b. Both experimental (Fig. 10a) and theoretical (Fig. $10 \mathrm{~b})$ curves for the Doppler spectrum width show the expected improvements with the OAB and WTB techniques. Nonetheless, these are not well matched, as there are considerable differences in the values of SDs. This could be attributed to the fact that the theoretical values are based on fitting a Gaussian spectral shape to data that is not always Gaussian, and the spectrum width estimator sensitivity to the spectral shape. In the case of the velocity SD, the two local minima are at approximate ranges (79 and $83.5 \mathrm{~km}$ ) where the spectrum width reaches local minima (Fig. 6) and the SDs of the reflectivity reach local maxima. This is because the lower spectrum width corresponds to higher autocorrelation in sample time, which produces smaller variance of ve- locity estimates (Doviak and Zrnić 1993). As the correlation increases, $L_{I}$ decreases, but the effect in the case of velocity estimates is a net decrease in the variance. The $\operatorname{SD}(v)$ for both oversampling and averaging, and oversampling and whitening satisfies the WSR-88D requirements (less than $1 \mathrm{~m} \mathrm{~s}^{-1}$ ). The same requirement on the $\operatorname{SD}(\sigma)$ is fully met only if oversampling and averaging is applied.

Reduction in SDs for $L=9$ is presented next (Figs. 11,12 , and 13). Changes due to advection and evolution were comparable to the ones in Figs. 4, 5 and 6 but more pronounced. This is expected, as the time to collect data was $186 \mathrm{~s}$ (cf. $111 \mathrm{~s}$ for $L=3$ ). Data were collected on the same day but from a different location in the storm and there was about $15 \mathrm{~min}$ of time difference between the two sets of records. The $1-\mathrm{dB}$ error requirement for the reflectivity factor is met only in the case of oversampled and whitened data. In this example, the requirements for the velocity and spectrum width estimates are met with all three techniques. This is no surprise as the reflectivity is most stringent and dictates the dwell time. The improvement in spectral moment estimates due to whitening is more pronounced in the long pulse mode because the oversampling factor $(L=$ 9) is three times larger than in the short pulse mode. Observe that the theoretically obtained SDs are somewhat smaller than the experimental ones. This is likely due to the increased pulse depth (in range) of $750 \mathrm{~m}$, hence, further departure from the assumption of uniform reflectivity within the pulse, resulting in incomplete whitening (Torres and Zrnić 2003).

Scatterplots (Figs. 14, 15, and 16) of the OAB versus WTB estimates show significantly larger dispersion of results in the case of spectrum width (even though negative values were approximated with the adjacent nonnegative estimates). The overplot line is the best straight-line fit in the least mean squares sense and is obtained by minimizing the perpendicular offsets. In case of reflectivity and velocity, the fit is well balanced and shows no bias of the WTB estimates. The spectrum width fit exhibits no consistent bias but is slightly tilted in the counter clockwise direction. This effect along with the higher dispersion could be caused by the previously mentioned sensitivity of the spectrum width estimator to the non-Gaussian shape of the weather signal spectra that occurs in nature.

Finally, a comparison of variances of the estimates obtained by the three procedures and for the short pulse 
$(L=3)$ is summarized in Table 1 . The theoretical values [based on Eqs. (6.10), (6.21), and (6.30a) in Doviak and Zrnić (1993)] are also included. The variances have been obtained by averaging in both range (over 99 range locations) and time (1280 pulses or radials). Overall, the measured (in odd columns) and theoretical (even columns) ratios of variances agree very well, and the variances from whitened data are smallest.

\section{Conclusions}

Definite demonstration of a novel procedure to process weather radar signals has been made. It was accomplished on actual time series data recorded on the NOAA research and development radar while the antenna was stationary. This radar is an augmented WSR$88 \mathrm{D}$. For this paper, the pertinent addition is a commercial processor capable of sampling radar signals several times per pulse duration.

Processing consisted of decorrelating the oversampled signals in range and applying standard procedures to the whitened samples. The standard deviations of whitening-based estimates were compared to the ones obtained by regular (matched filter) processing and to the ones from simple averages of autocovariances. Variance reduction equal to the number of oversampled points was achieved with the novel scheme as predicted by the theory. This verification was done on weather signals with large $(>35 \mathrm{~dB})$ SNR.

The implications of this conceptual proof could be far reaching. Because errors of estimates are inversely proportional to the volume scanning times, it follows that weather phenomena can be surveyed faster, while maintaining the same level of accuracy as present processing methods. Alternatively, variance of estimates can be improved at the current volume scanning times. The method has no requirements for transmitter bandwidth other than the usual ones for pulse Doppler radars, and it allows traditional processing (i.e., matched filter), which is advantageous at low SNRs. Further, the tests demonstrate that the technique is completely compatible with the WSR-88D and thus could be implemented on the U.S. national network of weather radars (Torres and Zrnić 2002).

Acknowledgments. The WSR-88D research radar is maintained by Mike Schmidt and Richard Wahkinney.
Dr. Valery Melnikov was instrumental for data collection, recording, and manipulation of the RVP7 processor. The National Weather Service has and continues to provide help in maintaining the radar, and the Radar Operation Center of the NWS was responsible for installing and supporting the RVP7 processor. Dr. Richard Passarelli and Mr. Alan Sigia of SIGMET gave us valuable guidance concerning the intricacies of the RVP7 processor. Funding for this research was provided under NOAA-OU Cooperative Agreement NA17RJ1227 and in part by the Federal Aviation Agency through Interagency Agreement DTFA 03-01-X9007.

\section{REFERENCES}

Doviak, R. J., and D. S. Zrnić, 1993: Doppler radar and weather observations. Academic Press, $562 \mathrm{pp}$.

- - - , and A. Shapiro, 2001: Phased array weather radarBenefits and challenges. Preprints, 30th Int. Conf. on Radar Meteorology, Munich, Germany, Amer. Meteor. Soc., 202-204.

Fabry, F., A. Bellon, M. R. Duncan, and G. L. Austin, 1994: High resolution rainfall measurement by radar for very small basins: The sampling problem reexamined. J. Hydrol., 161, 415-428.

Koivunen, A. C., and A. B. Kostinski, 1999: The feasibility of data whitening to improve performance of weather radar. J. Appl. Meteor., 38, 741-749.

Kostinski, A. B., and A. C. Koivunen, 2000: On the condition number of Gaussian sample-covariance matrices. IEEE Trans. Geosci. Remote Sens., 38, 329-332.

Owen, H., H. Urkowitz, J. Melody, J. Stephans, J. Kanefield, and S. Katz, 1998: Collection and processing of environmental data using a tactical shipboard radar. Preprints, 14th Int. Conf. on Interactive Information and Processing Systems (IIPS) for Meteorology, Oceanography and Hydrology, Phoenix, AZ, Amer. Meteor. Soc., 316-120.

Schultz, J. T., and A. B. Kostinski, 1997: Variance bounds on the estimation of reflectivity and polarization parameters in radar meteorology. IEEE Trans. Geosci. Remote Sens., 35, 248-255.

Shapiro, A., P. Robinson, and J. Wurman, 2001: Single-Doppler velocity retrievals with rapid-scan data. Preprints, 30th Int. Conf. on Radar Meteorology, Munich, Germany, Amer. Meteor. Soc., 194-196.

SIGMET, 1999: RVP7 digital receiver and Doppler signal processor user's manual. SIGMET, $212 \mathrm{pp}$.

Torres, S. M., 2001: Estimation of Doppler and polarimetric variables for weather radar. Ph.D. dissertation, The University of Oklahoma, $158 \mathrm{pp}$.

_- and D. S. Zrnić, 2003: Whitening in range to improve weather radar spectral moment estimates. Part I: Formulation and simulation. J. Atmos. Oceanic Technol., 20, 1433-1448.

Zrnić, D. S., and S. M. Torres, 2002: Efficient estimation of spectral moments and the polarimetric variables on weather radars, sonars, sodars, acoustic flow meters, lidars, and similar active remote sensing instruments. U.S. patent 6,448,923 B1. 